\title{
Experimental and numerical investigation of flutter phenomenon of an aitcraft wing (NACA 0012)
}

\author{
Tawfiq Khatir*, Boutchicha Djillali**, Samir Khatir***, Lousdad Abdelkader****, \\ Magd Abdel Wahab***** \\ *Institute of science and technology University Centre Salhi Ahmed, Naama 45000, Algeria \\ **Mechanical Engineering Department, University of Sciences and Technology of Oran Mohamed Boudiaf (USTO-MB), \\ BP1505 Oran El M'Naouer 31000, Algeria \\ ***Institute of science and technology University Centre Salhi Ahmed, Naama 45000, Algeria \\ ****Laboratory Mechanics of Structures and Solids (LMSS) Mechanical Engineering Department-Faculty of Technology, \\ University of Sidi Bel Abbès BP 89, Cité Ben M’ hidi Sidi Bel-Abbes 22000, Algeria \\ *****Division of Computational Mechanics, Ton Duc Thang University, Ho Chi Minh City, Vietnam; Faculty of Civil En- \\ gineering, Ton Duc Thang University, Ho Chi Minh City, Vietnam; Soete Laboratory, Faculty of Engineering and Archi- \\ tecture, Ghent University, Technologiepark Zwijnaarde 903, B-9052 Zwijnaarde, Belgium, \\ E-mail: magd.abdelwahab@tdt.edu.vn;magd.abdelwahab@ugent.be
}

cross $^{\text {ref }}$ http://dx.doi.org/10.5755/j01.mech.23.4.15175

\section{Introduction}

The floating is a phenomena generally observed in structures subjected to fluid flow, which is described by fluid-structure interaction. It considers the phenomenon of control surface flutter and the effect of rigid body modes [1]. The vibration of a NACA 0015 airfoil model with pitch and plunge degrees of freedom is investigated in a high-speed wind tunnel using motion sensors, pressure sensors on the airfoil surface and synchronized high-speed Schlieren visualizations of the unsteady flow field at Reynolds numbers and Compared with other studies [2].

The importance of flow and boundary layer control and adaptive of wing has revolutionary new benefits for take-off and landing conditions for many aircrafts $[3,4]$. It enhances the structural vibrations and reduces the possibility of damage in the structure. Furthermore, the phenomenon of "flutter" is dangerous leading to degradation of the structure. The wing and fuselage of an aircraft can be exposed to flutter. The first case of floating has been documented in 1916 and it was concerned with the bombardier Handley. The observed floating implicated an opposition mode of phase oscillation of left and right rudders coupled with a torsional mode of the fuselage (with amplitude of up to $45^{\circ}$ ); both of them at low frequencies [5]. The development of aircraft industries becomes a great deal of interest, when there is an advancement in adaptive structures and smart materials [6]. A model of NACA0012 benchmark experimental flutter results with unsteady pressure distributions were reported in the literature [7]. The aero-elastic behavior of rectangular wing was observed experimentally using acceleration and pressure and the wing was set at different angles and wind tunnel airspeeds [8]. Reference [9] gives a general vision about investigation of flutter phenomenon in airplanes wings, specifically on planes of the aero design, which ones usually disregards this kind of study.

The torsional stiffness of the structure has an effect on the critical speed of flexion-torsion flutter. The flexural stiffness of the wing is considered to be insignificant, even though it influences the critical speed of the flutter. The in- plane shape of the wing influences the aerodynamic, elastic and inertial characteristics of the wing, through which it influences the critical speed of the flutter. The position of the center of gravity of the wing has a considerable effect on the value of the critical speed of the flexion-torsion flutter. With the increase in flight altitudes, the speed of the flutter increases.

Let's consider an aircraft wing with length $l$ as shown in Fig. 1, from physical point of view, it is wise to simplify the representation of its behavior by using the concept of typical section.

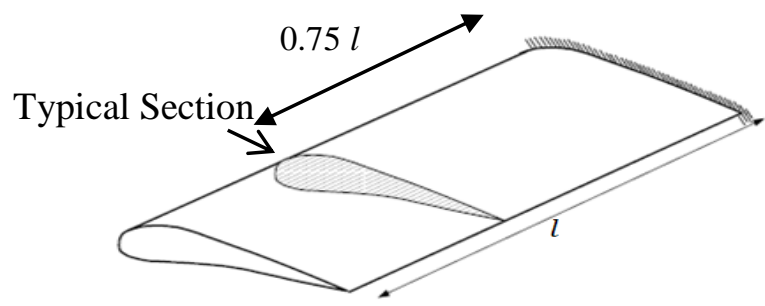

Fig. 1 Choice of a typical section of an aircraft wing

The typical section is a two-dimensional model representing the aerodynamic (profile) and elastic (flexion and torsion) properties of the wing for the study and analysis of aero-elastic behavior.

The first aero-elastic researchers (1920-1935) had observed that the choice of the section located at $75 \%$ of the distance between the joint and the end of the wing gave good results for the correlation between the theory and experiment. We can also approach this simplified model using a typical experimental configuration of fan tests, which study the response of wing segment with constant section submerged in a uniform flow and linked to the walls by flexible springs. In the two-dimensional study of aero elastic phenomena, we use the model illustrated in Fig. 2 [10].

In the model presented in Fig. 2, the aerodynamic profile is assumed to be that of the wing at the chosen location. 
The three characteristic points of the profile are: 1) the aerodynamic center or focal point, located at a relative distance $x_{a c}$ from the edge to which the lift $L$ is applied, 2) the elastic center, located at a relative distance $\left(x_{0}-x_{a c}\right)$ from the aerodynamic center and 3 ) the center of gravity $G$, located at a distance $\left(x_{c g}-x_{a c}\right)$ from the aerodynamic center. The attack angle $\alpha$ is positive in the upper direction (swing movement), whereas the deflection $h$ is positively measured in the downward direction.

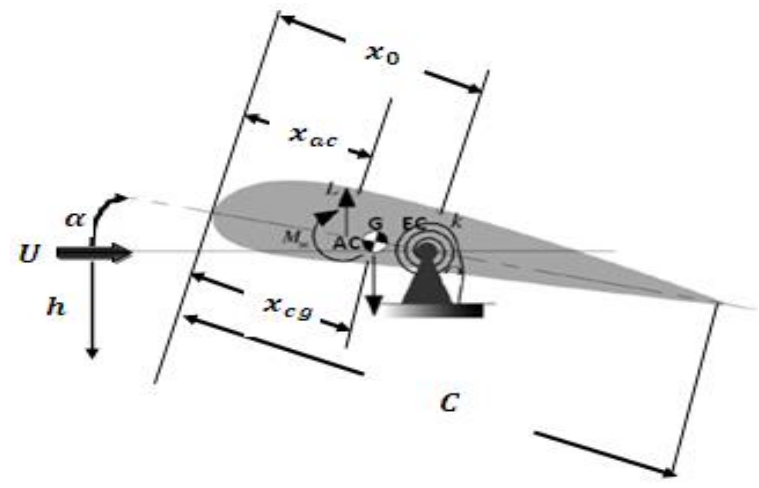

Fig. 2 Two-dimensional model of a typical section

The flutter designates the non-damped oscillations of some construction parts under the action of aerodynamic forces that are generated during oscillations. This type of oscillations appears to be automatically excited and is governed by the aerodynamic forces. The required energy for maintaining the oscillations is consumed by the oscillating system from the flow of air. The geometric properties of the wing profile used in this study are given as follows: area $=2847.52 \mathrm{~mm}^{2}$ and center of gravity with respect to global system of coordinates: $\mathrm{x}=0, \mathrm{y}=0, \mathrm{z}=-66.79 \mathrm{~mm}$.

Using experimental dynamic tests and numerical analysis, the phenomena of fluid-structure interaction is investigated. In this regards, we have conducted experimental tests aiming to study the dynamic behavior of a wing profile with respect to the speed of flow for different incident angles. The wing profile was elastically fixed from its extremities and dipped in a flow of air in a subsonic fan. We recorded the amplitudes of the movements by means of a spectrum analyzer. Furthermore, Finite Element Analysis is conducted and the numerical results are compared to those obtained from experiments.

\section{Experimental dynamic tests}

Due to the size of the structure, it is possible to consider an experimental dynamic study of the wing profile carried out in a laboratory. Such approach is made possible by using an accelerometer (a sensor). The excitation signal and the vibratory response are simultaneously measured using a spectrum analyzer, which allows the estimation of the modal parameters of the structure through the transfer functions measured at different points of the system. The excitation is made by the air flow of the fan .

The experimental bench was supported by a frame on which the structure is located as shown in Fig. 3. This structure has four flat knives placed horizontally in the same direction of the air flow generated by the fan. These knives are fixed at their ends by four squared supports. Two of these supports are fixed to the frame and the other two supports are supporting the wing in the horizontal position and perpendicular to the air flow with respect to the ground. At the other end of the structure, a sensor has been attached in the horizontal direction and perpendicular to the flow. The tests have been carried out on the wing profile by the variation of the speed of the air flow. To change the speed, we relied on the variation of pressure indicator in Pitot's tube. The range of pressure varies from zero to the limit pressure (return flow) and by varying the incident angle from $0^{\circ}$ to $15^{\circ}$ with $5^{\circ}$ increment.

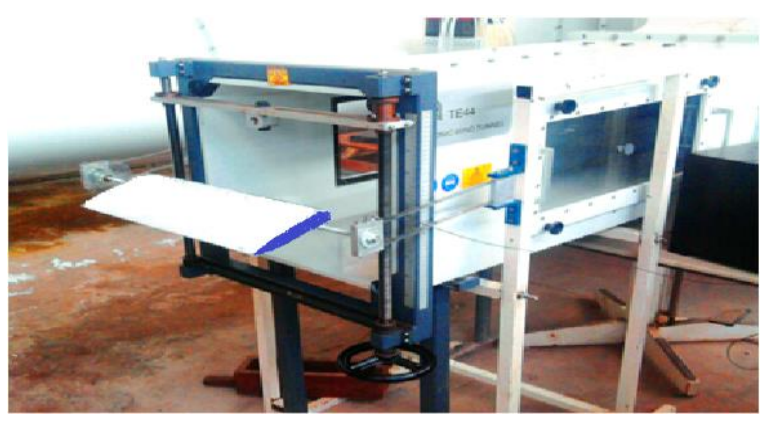

Fig. 3 Experimental bench

We have studied the effect of the speed of the wind on the vibrations of the aircraft wing. The frequency spectrums have been recorded in the range from 0 to $25 \mathrm{~Hz}$. The experimental normal frequencies are obtained directly from the analyzer as pic abscissa in the frequency domain response. Fig. 4 shows an example of the results of the measurements carried out on the wing profile in the fan by the spectrum analyzer at flow speed of $13.132 \mathrm{~m} / \mathrm{s}$ and incident angle of $\alpha=0^{\circ}$.

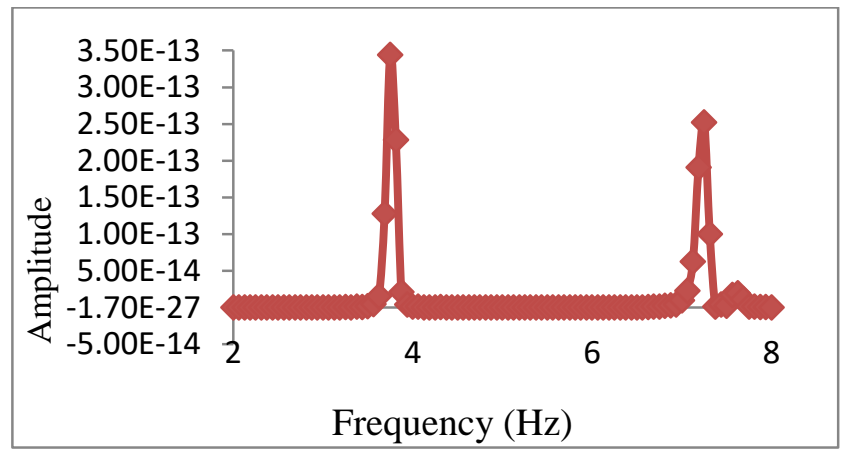

Fig. 4 Variation of the amplitudes with respect to the frequencies

The analysis of the frequency measurements was carried out made via CVP file using a Microsoft Excel matrix. The frequency domain plots of amplitude versus frequency were in the range of 0 to $25 \mathrm{~Hz}$. The complete numerical results for different speeds and angles of attack are listed in Tables 1, 2 and 3.

Figs. 5 to 7 show the influence of the speed on the amplitude of the first two modes of vibrations. These experimental results confirm that the amplitude increases progressively with respect to the speed for all considered incident angles. By comparing the amplitudes of modes 1 and 2, it is observed that mode 1 , which corresponds to the bending vibration, reach lower values of amplitude than mode 2 , which corresponds to torsional vibration. 
Table 1

Effect of the speed on and the first two natural frequencies and their amplitudes for $\alpha=0^{\circ}$

\begin{tabular}{|c|c|c|c|c|}
\hline Speed, $\mathrm{m} / \mathrm{s}$ & $f_{1}, \mathrm{~Hz}$ & $A_{1}$ & $f_{2}, \mathrm{~Hz}$ & $A_{2}$ \\
\hline 13.132 & 3.75 & 3.00 & 7.25 & 4.94 \\
\hline 18.572 & 3.87 & 4.41 & 7.25 & 8.11 \\
\hline 21.579 & 3.93 & 4.69 & 7.25 & 9.46 \\
\hline 24.568 & 3.81 & 6.32 & 7.18 & 14.5 \\
\hline 27.858 & 3.87 & 8.39 & 7.25 & 18.0 \\
\hline 31.627 & 4.00 & 7.50 & 7.25 & 15.9 \\
\hline 34.745 & 4.06 & 6.10 & 7.25 & 14.1 \\
\hline 37.375 & 3.87 & 5.40 & 7.25 & 16.4 \\
\hline
\end{tabular}

Table 2

Effect of the speed on the first two natural frequencies and their amplitudes for $\alpha=10^{\circ}$

\begin{tabular}{|c|c|c|c|c|}
\hline Speed, $\mathrm{m} / \mathrm{s}$ & $f_{1}, \mathrm{~Hz}$ & $A_{1}$ & $f_{2}, \mathrm{~Hz}$ & $A_{2}$ \\
\hline 14.38 & 3.68 & 6.58 & 7.18 & 14.21 \\
\hline 17.61 & 3.62 & 7.87 & 7.18 & 13.72 \\
\hline 20.76 & 3.62 & 12.21 & 7.06 & 17.41 \\
\hline 23.85 & 3.56 & 22.02 & 7.06 & 31.53 \\
\hline
\end{tabular}

Table 3

Effect of the speed on the first two natural frequencies and their amplitudes for $\alpha=15^{\circ}$

\begin{tabular}{|c|c|c|c|c|}
\hline Speed, $\mathrm{m} / \mathrm{s}$ & $f_{1}, \mathrm{~Hz}$ & $A_{1}$ & $f_{2}, \mathrm{~Hz}$ & $A_{2}$ \\
\hline 13.13 & 3.68 & 4.49 & 7.06 & 13.50 \\
\hline 16.08 & 3.68 & 17.40 & 7.07 & 26.23 \\
\hline 17.61 & 3.68 & 15.31 & 7.06 & 30.50 \\
\hline 19.03 & 3.68 & 12.61 & 7.06 & 27.36 \\
\hline 20.76 & 3.68 & 11.23 & 7.06 & 25.03 \\
\hline 23.85 & 3.62 & 14.52 & 7.06 & 22.22 \\
\hline 28.77 & 3.75 & 16.23 & 7.18 & 25.33 \\
\hline 30.23 & 3.75 & 18.01 & 7.18 & 24.90 \\
\hline
\end{tabular}

\section{Numerical analysis}

All the numerical results in this section are obtain using the finite element code ANSYS CFX WORKBENCH version 11. ANSYS CFX is a computational fluid dynamics software, which can be used to simulate the interaction between the wing and the wind produced by the fan in the experimental chamber. The main module used for ANAYS CFX is illustrated in Fig. 8. In a first step, the NACA Airfoil profile is imported to ANSYS and a 3D solid of the wing is created. Next, the air in the chamber surrounding the wing is modelled with a $3 \mathrm{D}$ prism. Then, the airfoil solid is subtracted from the prism to produce a new volume. This new volume is meshed with 3D tetrahedron 10-noded elements as shown in Fig. 9. In this model, the total number of nodes is 455698 and the total number of elements is 71365 . In our case study the results are limited to natural frequencies of the system and modal deformation associated to each mode, as well as, the determination of the critical speed.

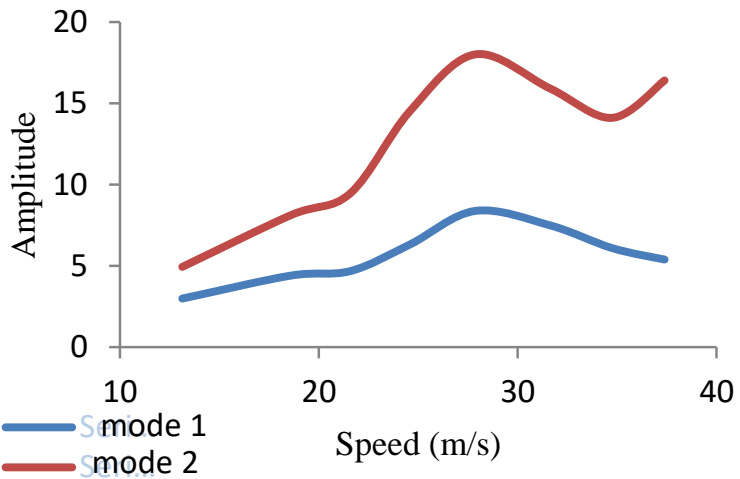

Fig. 5 Experimental determination of the speed versus the amplitudes of the first two modes of vibration for $\alpha=0^{\circ}$

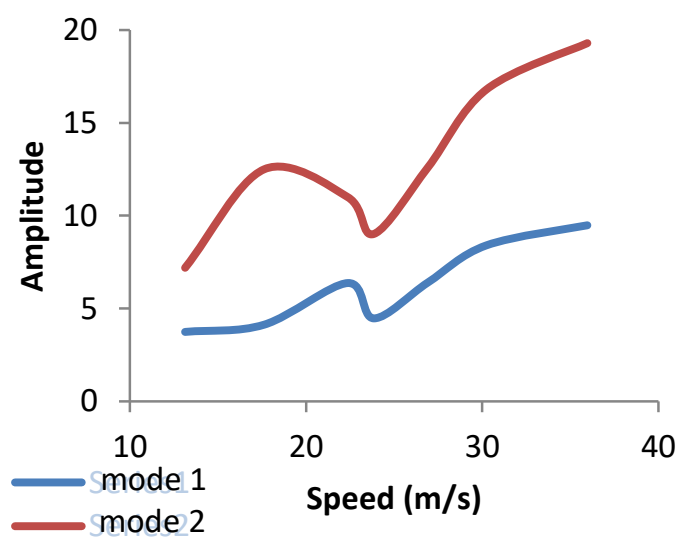

Fig. 6 Experimental determination of the speed versus the amplitudes of the first two modes of vibration for $\alpha=10^{\circ}$

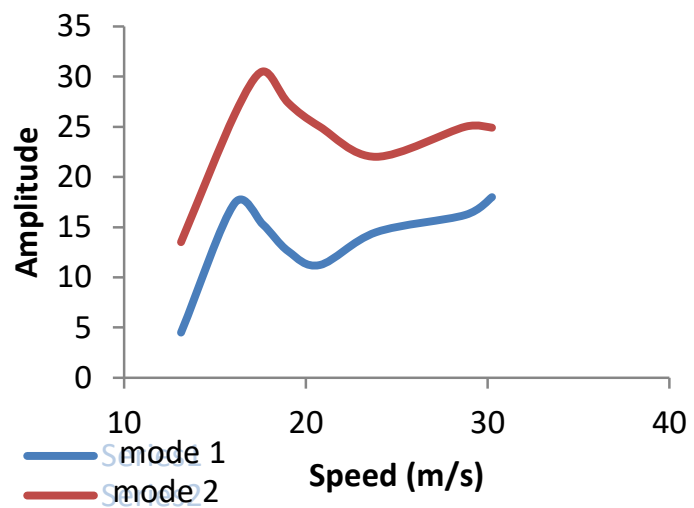

Fig. 7 Experimental determination of the speed versus the amplitudes of the first two modes of vibration for $\alpha=15^{\circ}$

The numerical predicted speed versus amplitudes of the first two modes of vibration is plotted in Figs. 10 to 12 for the first two modes of vibrations and for different angels of attach. Comparing Figs. 10 to 12 with Figs. 5 to 7, it can be seen that the numerically predicted curves have the same trends as the measured ones. The numerically predicted critical speed are also in good agreement with those measured experimentally. Therefore, the numerical model 


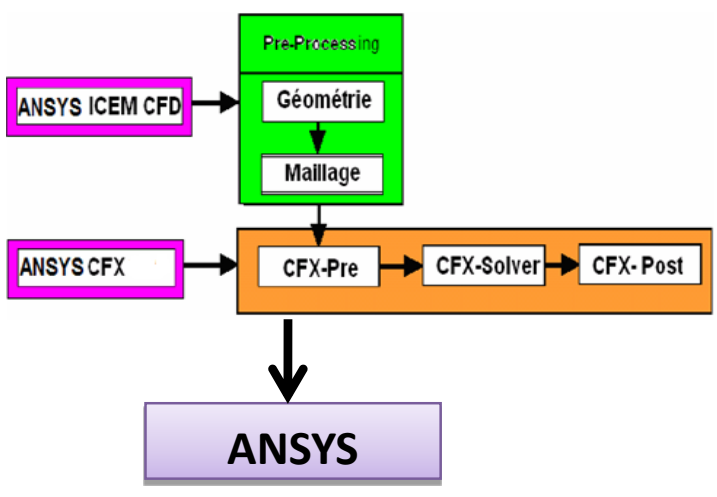

Fig. 8 Main modules CFX 11.0

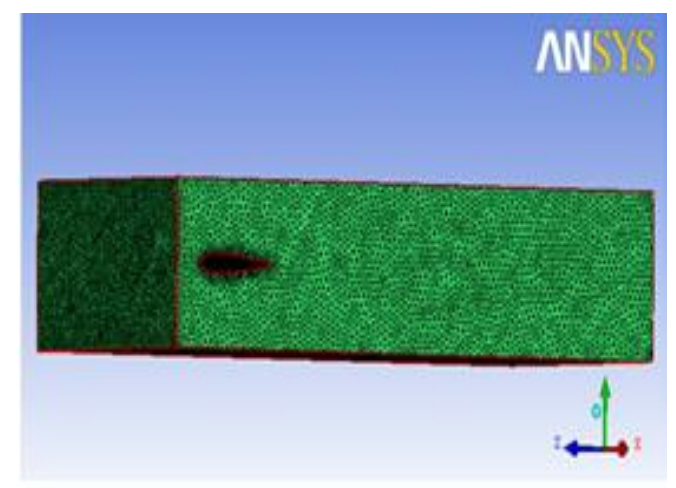

Fig. 9 Mesh representation in the studied zone

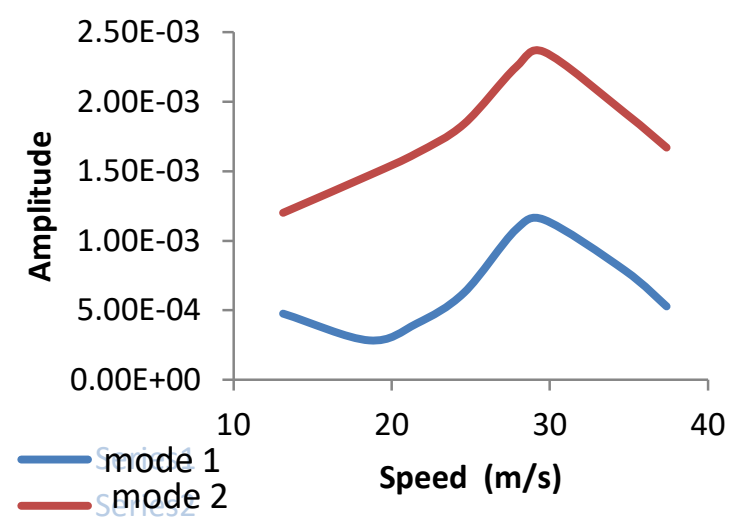

Fig. 10 Numerical prediction of the speed versus amplitudes of the first two modes of vibration for $\alpha=0^{\circ}$

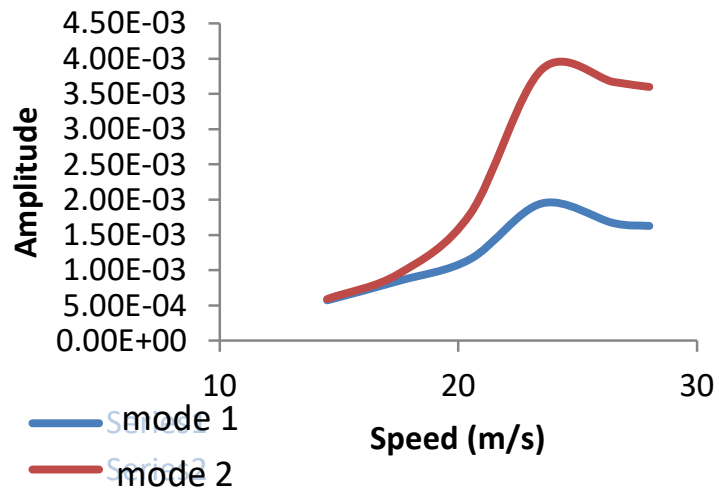

Fig. 11 Numerical prediction of the speed versus amplitudes of the first two modes of vibration for $\alpha=10^{\circ}$

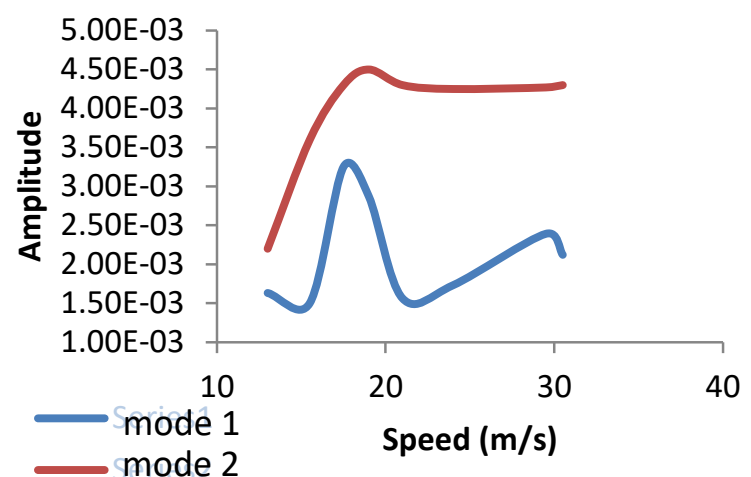

Fig. 12 Numerical prediction of the speed versus amplitudes of the first two modes of vibration for $\alpha=15^{\circ}$

can be used with some confidence to predict the aerodynamics behavior of the aircraft wing. From the results, it can also be seen that by increasing the angle of attack, the critical speed and the natural frequencies are reduced.

The results of the numerically calculated frequencies with those obtained by experiments, as well as, the deviation of each mode are reported in table 4 . By comparing the numerical and experimental results of the first two natural frequencies, a good agreement is observed. A numerical error below $3 \%$ is considered as satisfactory. The difference can be attributed to the errors generated during the experiments and the wing geometry, which might not be constant through its entire length during construction, i.e. symmetry of intrados and extrados. Furthermore,

Table 4 Calculated and measured results of natural frequencies for the first two modes

\begin{tabular}{|c|c|c|c|}
\hline \multirow{2}{*}{ Modes } & \multicolumn{3}{|c|}{ Frequencies (Hz) } \\
\cline { 2 - 4 } & Experimental & Numerical & Dev., \% \\
\hline 1 & 3.75 & 3.86 & 2.93 \\
\hline 2 & 7.25 & 7.23 & 0.27 \\
\hline
\end{tabular}

\section{Conclusion}

In this study, the floating critical speed of an aircraft wing in a subsonic fan was determined using dynamic experimental tests and numerical simulations. The experiments were conducted using a laboratory test set-up and by generating air flow using a fan. The commercial Finite Element Analysis package ANSYS CFX was used for the numerical simulations. In general, the results obtained experimentally are in good agreement with those obtained using the numerical simulations. From our study, the following conclusions are drawn:

- The amplitude of the second mode is more important than that of the first mode.

- If the inclination increases, the flutter critical speed decreases.

- Above the critical speed, the amplitudes of vibrations decrease and the profile changes when a certain speed is reached. 


\section{References}

1 Dynamic Aeroelasticity - Flutter. 2015. Introduction to Aircraft Aeroelasticity and Loads: 171-205. http://dx.doi.org/10.1002/9781118700440.ch10.

2 Šidlof, P.; Vlček, V.; \& Štěpán, M. 2016. Experimental investigation of flow-induced vibration of a pitch-plunge NACA 0015 airfoil under deep dynamic stall. Journal of Fluids and Structures 67: 48-59. http://dx.doi.org/10.1016/j.jfluidstructs.2016.08.011.

3 Thill, C.; Etches, J.; Bond, I.; Potter, K.; Weaver, P. 2008. Morphing Skins, The Aeronautical Journal 188: 128. http://dx.doi.org/10.1017/s0001924000002062.

4 Abbasi Hoseini, A., \& Masdari, M. 2004. Reducing Wind Tunnel Data for Flowfield Study over the WingCanard Configuration Using Neural Network. 42nd AIAA Aerospace Sciences Meeting and Exhibit. http://dx.doi.org/10.2514/6.2004-727.

5 Hodges, D. H., \& Pierce, G. A. 2011. Introduction to structural dynamics and aeroelasticity (Vol. 15). Cambridge University Press. http://dx.doi.org/10.1017/cbo9780511997112.006.

6 Mamou, M.; Mebarki, Y.; Khalid, M.; Genest, M. 2010. Aerodynamic performance optimization of a wind tunnel morphing wing model subject to various cruise flow conditions, in Proc of ICAS'27, paper 496: -18.

7 Rivera, JR.; Dansberry, B.; Bennett, R.; Durham, M.; Silva, W. 1992. NACA 0012 benchmark model experimental flutter results with unsteadypressure distributions. 33rd Structures, Structural Dynamics and Materials Conference. http://dx.doi.org/10.2514/6.1992-2396.

8 Razak, N. A.; Andrianne, T.; Dimitriadis, G. 2011. Flutter and stall flutter of a rectangular wing in a wind tunnel. AIAA Journal 49(10): 2258-2271. http://dx.doi.org/10.2514/1.j051041.
9 Dal'Carobo, B.; Fensterseifer, R. 2010. Aircraft Wing Flutter: An Applied Approach. SAE Technical Paper Series. http://dx.doi.org/10.4271/2010-36-0513.

10 Shirk, M. H.; Hertz, T. J.; Weisshaar, T. A. 1986. Aeroelastic tailoring-theory, practice, and promise. Journal of Aircraft 23(1): 6-18. http://dx.doi.org/10.2514/3.45260.

T. Khatir , B. Djillali, S. Khatir, A. Lousdad and M. Abdel Wahab

\section{EXPERIMENTAL AND NUMERICAL INVESTIGA- TION OF FLUTTER PHENOMENON OF AN AITCRAFT WING (NACA 0012)}

S u m m a r y

Floating is a dangerous phenomenon for aircrafts and usually ends with plane rupture. It can be used to characterize a dynamic instability of the structure. Floating phenomenon is usually due to an interaction between aerodynamics, elasticity and inertial forces. It appears at a determinate speed of flight called critical floating speed. The aim of the present study is to determine the floating critical speed of an aircraft wing in a subsonic fan by means of experimental tests and numerical simulations using the commercial Finite Element Analysis package ANSYS CFX. The obtained experimental results are in good agreement with those obtained using the numerical approach.

Keywords: Aerodynamics, Floating, Flutter phenomenon, Aircraft wing, Finite Element Analysis, Computational Fluid Dynamics.

Received June 06, 2016 Accepted August 04, 2017 